\title{
Instability of strongly charged sources in classical scalar electrodynamics
}

\author{
M. Bawin and J. Cugnon \\ Institut de Physique B5, Université de Liège, Sart Tilman, B-4000 Liège 1, Belgium \\ (Received 28 January 1983)
}

\begin{abstract}
We solve the equations of motion of classical scalar electrodynamics for a fixed source in electromagnetic interaction with a scalar field. We find that above some critical coupling strength there exist field configurations corresponding to a partial screening of the external source, which have a lower energy than the pure Coulomb (with zero scalar field) solution. Thus, in classical scalar electrodynamics, there is an upper limit on the charge of stable external sources.
\end{abstract}

\section{INTRODUCTION}

The problem of charge screening of an external source in electromagnetic interaction with a scalar field was recently studied by Klein and Rafelski ${ }^{1}$ and by Mandula ${ }^{2}$ in the case where the scalar particles are massless. While Mandula solved exactly the classical field equations, Klein and Rafelski derived self-consistent equations from a mean-field approximation to the quantum-field-theoretic problem. Physical interest in this problem is twofold. First, it provides a theoretical model to study pion condensation due to a priori known strong (albeit long-range Coulomb) interactions and may be of interest in connection with the problem of pion condensation in nuclei due to strong nuclear forces. Second, it has been suggested that this problem could be relevant to the question of quark confinement ${ }^{2-4}$ : the unobservability of quarks would then result from the instability of one-particle quark states. Our own motivation for the study of this problem lies more precisely in the question of stability of strongly charged nuclei. ${ }^{5,6}$ Specifically, we wish to investigate how the existence of massive charged scalar particles affects the stability of nuclei, taking only electromagnetic interactions into account. Like Mandula, ${ }^{2}$ we have chosen to solve exactly the classical field equations of (massive) scalar electrodynamics for an external source in electromagnetic interaction with a scalar field. Although our results cannot be directly compared to those of Klein and Rafelski, ${ }^{1}$ as the latter include a pion self-interaction and use other field-strength parameters, we nevertheless also find that, beyond some critical value of the external charge, partial screening of the external source (pion condensation) occurs. That critical value is exactly the value for which the Klein-Gordon equation for a scalar particle of mass $m$ in the external Coulomb field of the source yields bound eigenstates of energy $\omega=-m$. These partial-screening configurations have a lower energy than the pure Coulomb (with zero pion field) configuration. This is to be contrasted with Mandula's results for massless pions, ${ }^{2}$ where total screening of the external charge occurs. We thus find that classical electrodynamics indeed puts an upper value on the charge of stable strongly charged sources.

Our paper is organized as follows. In Sec. II, we write the nonlinear classical equations of motion for a Coulomb source in electromagnetic interaction with a charged scalar field. Section III is devoted to a numerical study of these equations and to a discussion of our results.

\section{CLASSICAL FIELD EQUATIONS}

The Lagrangian density $\mathscr{L}$ describing the interaction of a charged boson field $\phi$ with the electromagnetic field $A^{\mu}$ in the presence of an externally prescribed current $j_{\mu}^{\text {ext }}$ is given by ${ }^{7}$

$$
\begin{aligned}
\mathscr{L}= & \left(\partial_{\mu} \phi-i e A_{\mu} \phi\right)\left(\partial^{\mu} \phi^{*}+i e A^{\mu} \phi^{*}\right)-m^{2} \phi \phi^{*} \\
& -\frac{1}{4}\left(\partial_{\mu} A^{v}\right)\left(\partial^{\mu} A_{v}\right)-e j_{\mu}^{\mathrm{ext}} A^{\mu} .
\end{aligned}
$$

From (1), one gets the following equations of motion (we take $\left.\alpha \equiv e^{2} / 4 \pi=1 / 137\right)$ :

$$
\begin{aligned}
& \left(\partial_{\mu}-i e A_{\mu}\right)^{2} \phi+m^{2} \phi=0, \\
& \square A_{\mu}-\partial_{\mu} \partial^{v} A_{\nu}+i e\left(\phi^{*} \overleftrightarrow{\partial}_{\mu} \phi-2 i e A_{\mu} \phi^{*} \phi\right)=e j_{\mu}^{\text {ext }} .
\end{aligned}
$$

Taking now

$$
j_{\mu}^{\mathrm{ext}}=\rho(r) \delta_{\mu 0} \equiv \frac{e Z \delta\left(r-r_{0}\right)}{r^{2}} \delta_{\mu 0},
$$

where $\rho(r)$ is a static prescribed charged distribution corresponding to a total charge $\mathrm{Ze}$ distributed on a shell of radius $r_{0}$, and working in the radiation gauge

$$
\vec{\nabla} \cdot \vec{A}=0,
$$

one can see that Eqs. (2) and (3) have the trivial Coulomb solution:

$$
\begin{aligned}
& A_{0}=\frac{e Z}{4 \pi r} \quad\left(r>r_{0}\right), \\
& A_{0}=\frac{e Z}{4 \pi a} \quad\left(r<r_{0}\right), \\
& \phi=\overrightarrow{\mathrm{A}}=0 .
\end{aligned}
$$

The energy density $T_{00}$ corresponding to $\mathscr{L}$ from Eq. (1) is given by

$$
\begin{gathered}
\left.T_{00}=|| i \frac{\partial}{\partial t}-e A_{0}\right]\left.\phi\right|^{2}+|\vec{\nabla} \phi|^{2} \\
+m^{2}|\phi|^{2}+\frac{1}{2}\left(\vec{\nabla} A_{0}\right)^{2} .
\end{gathered}
$$

We now wish to show that Eqs. (2) and (3) have solutions with $\phi \neq 0$ whose corresponding energy is lower than the energy corresponding to solutions (6) and (7) above some critical $Z$ value.

As in Ref. 2 we look for spherically symmetric solu- 
tions of the form

$$
\begin{aligned}
& \phi(r)=e^{i \omega t} \widetilde{\phi}(r), \\
& A_{0} \equiv A_{0}(r), \\
& \overrightarrow{\mathrm{A}}=0 .
\end{aligned}
$$

Upon writing

$$
\begin{aligned}
& e A_{0}+\omega=\frac{f}{r}, \\
& \widetilde{\phi}=\frac{g}{\sqrt{2} e r},
\end{aligned}
$$

Eqs. (2), (3), and (8) become, respectively,

$$
\begin{aligned}
& \frac{d^{2} g}{d r^{2}}+\frac{f^{2}-m^{2} r^{2}}{r^{2}} g=0, \\
& \frac{d^{2} f}{d r^{2}}-\frac{g^{2}}{r^{2}} f=-\frac{\alpha Z}{r_{0}} \delta\left(r-r_{0}\right), \\
& T_{00}=\frac{f^{2} g^{2}}{2 e^{2} r^{4}}+\frac{1}{2 e^{2}}\left[\left[\frac{g}{r}\right]^{\prime}\right]^{2}+\frac{m^{2}}{2 e^{2}} \frac{g^{2}}{r^{2}} \\
& \quad+\frac{1}{2 e^{2}}\left[\left[\frac{f}{r}\right]^{\prime}\right]^{2} .
\end{aligned}
$$

The total energy $E$ associated with $T_{00}$ is of course given by

$$
E=4 \pi \int_{0}^{\infty} T_{00} r^{2} d r .
$$

Note that Eqs. (13) and (14) do not really contain two independent parameters $m$ and $r_{0}$. The only (dimensionless) parameter is actually $m r_{0}$. This can be seen by using $x=m r$ as a new variable in Eqs. (13) and (14).

If we drop the nonlinear term $g^{2}$ in (14), then the radial Klein-Gordon equation is recovered from Eq. (13):

$$
\left[\frac{d^{2}}{d r^{2}}+\left(e A_{0}-\omega\right)^{2}-m^{2}\right) \widetilde{g}=0,
$$

where $A_{0}$ is given by Eq. (6).

Guided by the corresponding analysis in the massless case $^{2}$ and our knowledge of the solutions to the linearized Klein-Gordon equation, we look for solutions to (13) and (14) satisfying the boundary conditions

$$
\begin{aligned}
& f \underset{r \rightarrow \infty}{\simeq}-m r+\alpha Q, \\
& g \underset{r \rightarrow \infty}{\simeq} C \exp \left[-(8 m \alpha Q r)^{1 / 2}\right], \\
& f \underset{r \rightarrow 0}{\simeq} f_{1} r, \\
& g \underset{r \rightarrow 0}{\simeq} g_{1} r,
\end{aligned}
$$

where $f_{1}, g_{1}, Q$, and $C$ are arbitrary constants to be determined, $Q$ being the effective charge of the source. Equation (14) also requires

$$
\left.f^{\prime}\right|_{r=r_{0}+\epsilon}-\left.f^{\prime}\right|_{r=r_{0}-\epsilon}=-\frac{\alpha Z}{r_{0}} .
$$

Continuity of $f, g, g^{\prime}$ together with (22) will determine the value of the four arbitrary constants.

Our choice of $\omega=-m$ in (11) is determined by the fact

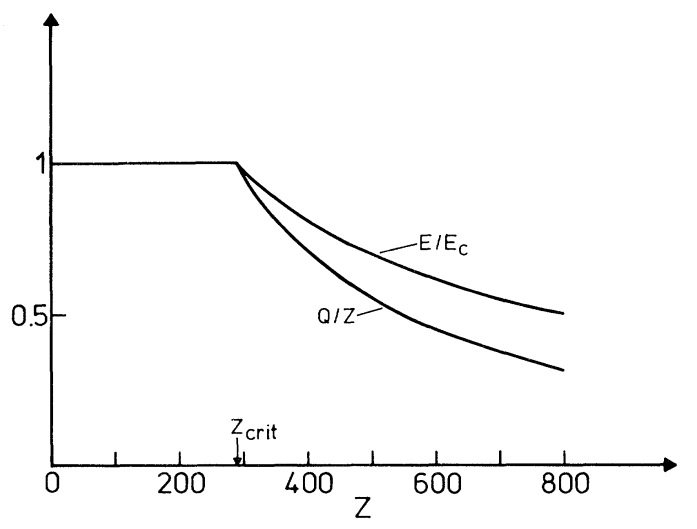

FIG. 1. Variation of the ratio $Q / Z$ as a function of $Z$; $e Q$ is the effective charge of the source while $e Z$ is the bare charge of the fixed source (i.e., in the absence of any scalar field). The other curve gives the ratio $E / E_{c}$ as a function of $Z ; E$ is the energy of the field while $E_{c}$ is the energy associated with a pure Coulomb solution. Both curves show that for $Z>Z_{\text {crit }}=290$ the partially screened solutions $(\boldsymbol{Q}<\boldsymbol{Z})$ have lower energy than the Coulomb solution.

that we expect a total breakdown of the validity of the one-particle interpretation of the Klein-Gordon equation for a $Z$ value $\equiv Z_{\text {crit }}$ such that $\omega=-m^{1}$. It is beyond the scope of this paper to study whether these solutions correspond to an absolute minimum of the field energy.

\section{RESULTS AND DISCUSSION}

As is well known, ${ }^{1}$ the Klein-Gordon equation (17) with $A_{0}$ given by (6) has complex eigenvalues for $Z>Z_{\text {crit }}$.

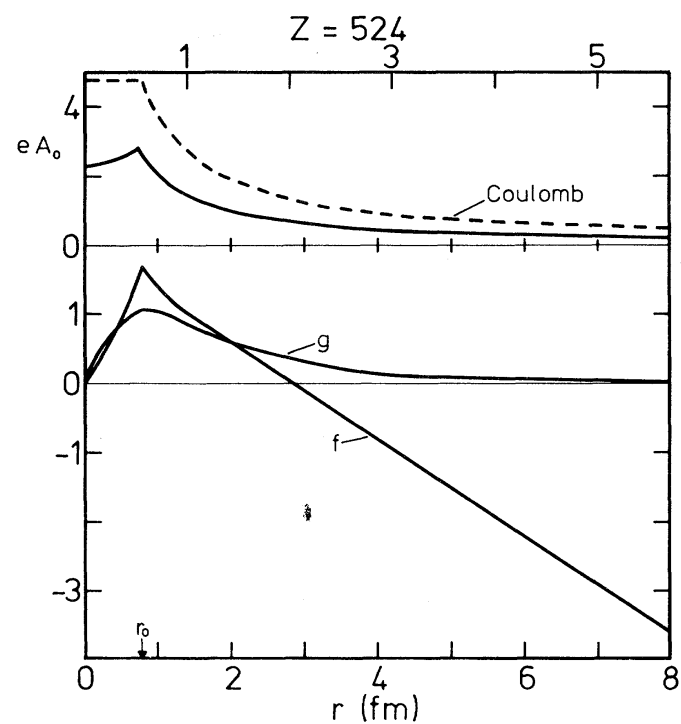

FIG. 2. Shape of the fields $f$ and $g$ in configuration space (lower part) for $Z=524$ ( $>Z_{\text {crit }}$ ). Their asymptotic behavior is as in Eqs. (18)-(21). The upper part gives the electric potential field $A_{0}$, which is related to $f$ through relation (11) (solid curve). The electric potential field corresponding to the pure Coulomb solution [Eq. (6)] is also shown for comparison. The scale on the top gives the distance $r$ in pion Compton wavelength units. 


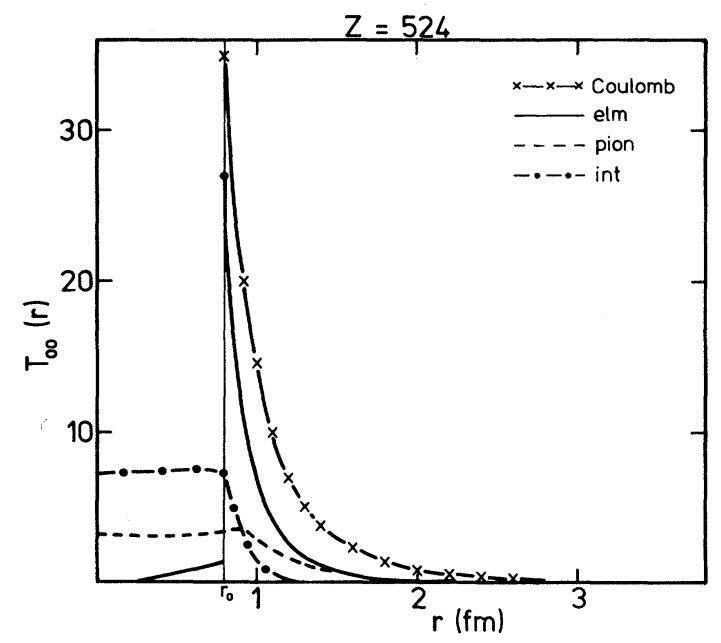

FIG. 3. Behavior of the various components to the energy density $T_{00}$ [Eqs. (25)-(27)], as a function of $r$, for $Z=524$. The energy density corresponding to the pure Coulomb solution [Eq. (6)] is shown for comparison. It vanishes for $r<r_{0}$.

Specifically, solving (17) in a standard way ${ }^{6}$ with $m$ equal to the pion mass $=139.6 \mathrm{MeV}$, we find

$$
Z_{\text {crit }}=290 \text {, }
$$

for $r_{0}=0.8 \mathrm{fm}$.

Our choice of $r_{0}$ turns out to be numerically convenient and has no deeper physical meaning.

Figure 1 shows our results for the total energy $E$ of the field as a function of the fixed charge $Z$ of the external source, together with the variation of the effective total charge $Q$ of the field (including the pion field) as a function of $Z$. As stated in Sec. II, the only parameter is $m r_{0}$, so that the ratio $E / E_{c}$ in Fig. 1 also holds for values of $r_{0}$ other than the one quoted, provided $m$ is changed in such a way that $m r_{0}$ remains constant. One can see that Eqs. (13) and (14) have solutions with $g \neq 0$ that have lower energy than the Coulomb solution $(g=0)$ for
$Z>Z_{\text {crit }} \equiv 290$. The remarkable feature of these solutions is that they correspond to a partial screening of the external source, as shown by curve 1 , in contrast with the corresponding result for massless pions, where total screening occurs. $^{2}$ Note that our result is in qualitative agreement with that of Klein and Rafelski ${ }^{1}$ who used a coherent-state approximation to the quantum-field-theoretic problem. Figure 2 illustrates the behavior of the solutions $f$ and $g$ for $Z=524>Z_{\text {crit }}$. We note that $f$ is practically linear in a wide range of $r$. In Fig. 3 we illustrate the importance of various components to the field energy $E$ by decomposing $T_{00}$ in the following way:

$$
T_{00}=T_{00}^{\mathrm{em}}+T_{00}^{\mathrm{pion}}+T_{00}^{\mathrm{int}},
$$

where

$$
\begin{aligned}
& T_{00}^{\text {pion }}=\left[\left[\frac{g}{r}\right]^{\prime}\right]^{2}+2 m^{2} \frac{g^{2}}{r^{2}}, \\
& T_{00}^{\mathrm{em}}=\left[\left[\frac{f}{r}\right]^{\prime}\right]^{2}, \\
& T_{00}^{\mathrm{int}}=\frac{f^{2} g^{2}}{r^{4}}-m^{2} \frac{g^{2}}{r^{2}} .
\end{aligned}
$$

This decomposition ensures that for $Z=0 T_{00}^{\text {pion }}$ is the free pion field energy density, while, for $g=0, T_{00}^{\mathrm{em}}$ is the energy density of the free electromagnetic field. $T_{00}^{\text {int }}$ then stands for the interaction energy density.

To conclude, we have found that classical massive scalar electrodynamics with a fixed external source has solutions corresponding to a partial screening of the source for values of the external charge larger than some critical value. These solutions have lower energy than the Coulomb solution, thus implying that, within classical electrodynamics, there exists an upper limit on the charge of stable external sources.

\section{ACKNOWLEDGMENT}

The work of one of us (M.B.) was supported by the Na. tional Fund for Scientific Research, Belgium.
${ }^{1}$ A. Klein and J. Rafelski, Z. Phys. A 284, 71 (1978). See also J. Rafelski, L. P. Fulcher, and A. Klein, Phys. Rep. 38C, 227 (1978).

2J. E. Mandula, Phys. Lett. 68B, 495 (1977).

${ }^{3}$ J. Finger, D. Horn, and J. E. Mandula, Phys. Rev. D 20, 3253 (1979).
4J. Rafelski, Phys. Lett. 79B, 419 (1978).

${ }^{5}$ P. Gärtner, B. Müller, J. Reinhardt, and W. Greiner, Phys. Lett. 95B, 181 (1980).

${ }^{6} \mathrm{M}$. Bawin and J. Cugnon, Phys. Lett. 107B, 257 (1981).

${ }^{7}$ See, e.g., S. Gasiorowicz, Elementary Particle Physics (Wiley, New York, 1966), p. 89. 